\title{
Room-Temperature Lasing in Colloidal Nanoplatelets via Mie- Resonant Bound States in the Continuum
}

\author{
Mengfei Wu, ${ }^{\perp}$ Son Tung Ha, ${ }^{\perp}$ Sushant Shendre, Emek G. Durmusoglu, Weon-Kyu Koh, \\ Diego R. Abujetas, José A. Sánchez-Gil, Ramón Paniagua-Domínguez, Hilmi Volkan Demir,* \\ and Arseniy I. Kuznetsov*
}

Cite This: Nano Lett. 2020, 20, 6005-6011

Read Online

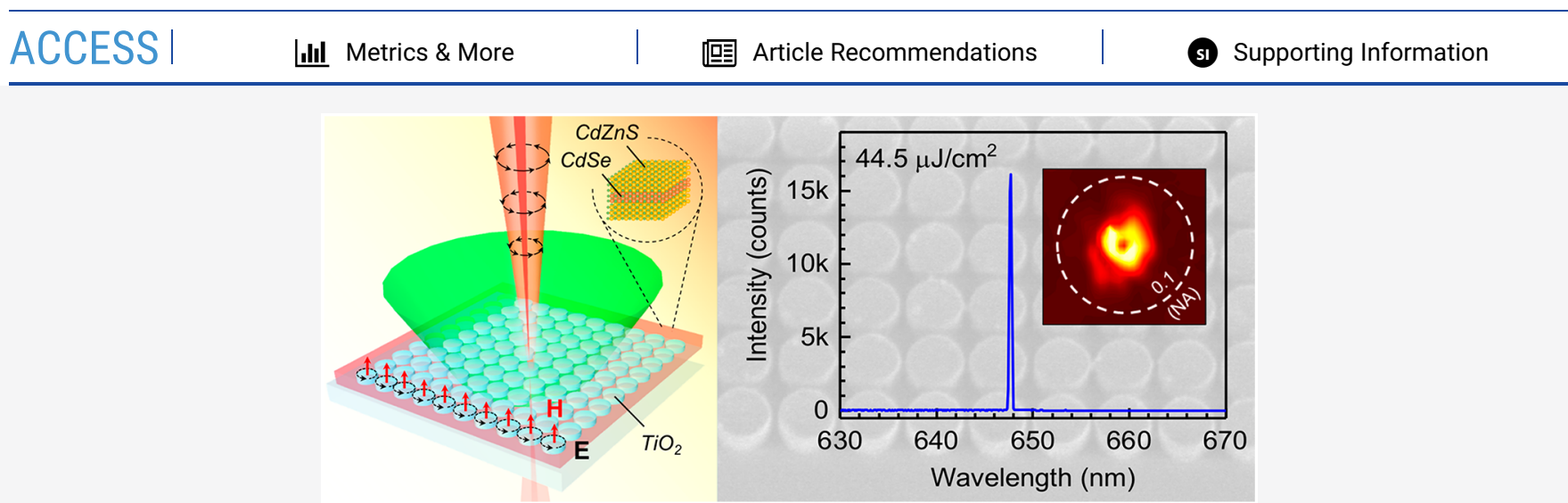

ABSTRACT: Solid-state room-temperature lasing with tunability in a wide range of wavelengths is desirable for many applications. To achieve this, besides an efficient gain material with a tunable emission wavelength, a high quality-factor optical cavity is essential. Here, we combine a film of colloidal $\mathrm{CdSe} / \mathrm{CdZnS}$ core-shell nanoplatelets with square arrays of nanocylinders made of titanium dioxide to achieve optically pumped lasing at visible wavelengths and room temperature. The all-dielectric arrays support bound states in the continuum (BICs), which result from lattice-mediated Mie resonances and boast infinite quality factors in theory. In particular, we demonstrate lasing from a BIC that originates from out-of-plane magnetic dipoles oscillating in phase. By adjusting the diameter of the cylinders, we tune the lasing wavelength across the gain bandwidth of the nanoplatelets. The spectral tunability of both the cavity resonance and nanoplatelet gain, together with efficient light confinement in BICs, promises low-threshold lasing with wide selectivity in wavelengths.

KEYWORDS: Mie resonances, bound states in the continuum, dielectric metasurfaces, colloidal nanoplatelets, room-temperature lasing

$\mathrm{E}$ fficient room-temperature lasers with a compact size and wide wavelength selectivity are highly desirable in biological imaging, near-field sensing, integrated photonics, tunable light sources, and advanced display technologies. ${ }^{1,2}$ One approach to realize such lasers is to integrate nanoscale photonic cavities with solution-processed gain materials that have a tunable gain spectrum. Examples include colloidal quantum dots in a distributed feedback (DFB) cavity, ${ }^{3}$ colloidal nanoplatelets in a photonic crystal, ${ }^{4}$ organic dyes in a $2 \mathrm{D}$ array of plasmonic nanoparticles, ${ }^{5,6}$ and each of these materials in a Fabry-Pérot cavity. $^{7-10}$ Among different types of cavities, the DFBs and plasmonic arrays rely on diffraction in a periodic structure to achieve sharp photonic modes ${ }^{11,12}$ and directional emission. ${ }^{13,14}$

Recently, periodic nanostructures made of dielectrics or semiconductors with high refractive indices $(n>2)$ have also been studied heavily. ${ }^{15-17}$ In contrast to plasmonic resonators, high-index dielectric nanostructures, with much lower losses at visible wavelengths, support not only electric multipoles but also magnetic multipoles. ${ }^{18,19}$ Coupling of these Mie resonances in a dielectric nanoparticle array gives rise to diffraction-mediated, high-quality $(Q)$-factor modes, ${ }^{20,21}$ among which are those known as bound states in the continuum (BICs) exhibiting extraordinarily high $Q$ factors. ${ }^{22-25}$ These are discrete states completely decoupled from the radiation field despite lying spectrally within a continuum of radiation modes. A BIC arises as a collective mode in a periodic system of nanoparticles supporting a multipolar mode, where the direction of any open radiation channel of the collective mode, as dictated by the Bloch wave vector, or the phase retardation between nanoparticles, coincides with a forbidden direction of radiation of the

Received: May 12, 2020

Revised: June 23, 2020

Published: June 25, 2020 

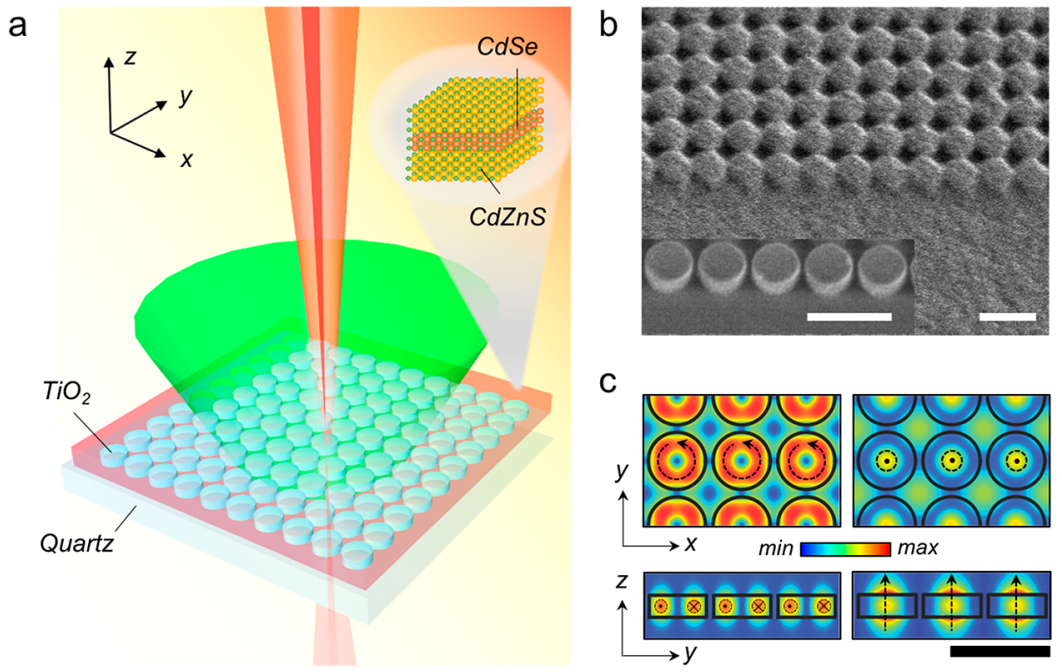

Figure 1. (a) Schematic of the device structure, optical excitation (green cone), and lasing output (red beam). The device consists of an array of $\mathrm{TiO}_{2}$ cylinders covered with spin-cast nanoplatelets (NPLs). Inset: $\mathrm{CdSe} / \mathrm{CdZnS}$ core-shell NPL. (b) SEM image of an array of TiO $\mathrm{O}_{2}$ cylinders coated with a layer of NPLs. Inset: individual $\mathrm{TiO}_{2}$ cylinders without NPLs. Scale bars: $500 \mathrm{~nm}$. (c) Electric field intensity in the cylinder array (diameter $D=300$ $\mathrm{nm}$, with $40 \mathrm{~nm}$ gap) for in-phase out-of-plane magnetic dipoles (left, $\lambda=625 \mathrm{~nm}$ ) and out-of-plane electric dipoles (right, $\lambda=548 \mathrm{~nm}$ ). Top: $x-y$ plane cut at half height; bottom: $y-z$ plane cut along the cylinder diameter. The direction of the electric field is indicated with dashed arrows. Black scale bar: $500 \mathrm{~nm}$.

constituent multipolar mode. For instance, the only radiation channel of a subdiffractive array of electric or magnetic dipoles (or any other multipoles) oscillating in phase would be in the direction normal to the lattice plane, where the waves interfere constructively. That is provided that the dipoles radiate energy in the out-of-plane direction. If they do not, as in the case of dipoles oscillating in the normal direction, no energy would be released from the system of in-phase dipoles, and a cavity is formed with an infinite $Q$ factor, in theory. In practice, due to a finite sample size, material absorption, and surface scattering, any BIC becomes a quasi-BIC with a finite but extremely high $Q$ factor. $^{22,23,26}$ The $Q$ factor is further reduced if the array is diffractive with a period large enough to support radiation at oblique angles for in-phase multipoles. In addition, breaking the in-plane inversion symmetry of the unit cell would also transform a BIC into a quasi-BIC. ${ }^{27-29}$ Using these concepts, fluorescence enhancement has been demonstrated in quasi-BIC cavities, ${ }^{30,31}$ while lasing based on BICs and quasi-BICs in periodic dielectric ${ }^{32-37}$ and metallic ${ }^{6,38-41}$ nanostructures has also been reported in recent years.

In line with these research efforts, our previous work demonstrated lasing in an array of gallium arsenide (GaAs) cylinders from a BIC comprising out-of-plane electric dipoles. ${ }^{36}$ Due to substantial nonradiative losses in GaAs, lasing was observed only at cryogenic temperatures. To achieve roomtemperature lasing, either the resonator needs to be made of a highly efficient gain material such as III-V multiple quantum wells, ${ }^{35}$ or a hybrid device structure has to be adopted, in which materials for the Mie resonator and the gain medium are independently optimized and closely integrated. In this study, we take the latter approach by depositing a thin film of colloidal nanoplatelets (NPLs) as the gain material on top of an array of titanium dioxide $\left(\mathrm{TiO}_{2}\right)$ cylinders supporting BICs. The NPLs are quantum wells synthesized in solutions, ${ }^{42}$ flat in $2 \mathrm{D}$ with charges confined in the third dimension, which is a few atomic layers thick. More specifically, the NPLs we use are composed of four monolayers of cadmium selenide ( $\mathrm{CdSe}$ ) core, ${ }^{43}$ sandwiched between cadmium zinc sulfide $(\mathrm{CdZnS})$ shells. ${ }^{44}$
The NPLs typically have a high optical gain, thanks to their low Auger recombination losses. ${ }^{45}$ Moreover, the gain wavelength can be readily tuned by changing the chemical composition or thickness of the NPLs. ${ }^{46}$ Optically pumped lasing at room temperature has been demonstrated in NPLs across the visible spectrum in Fabry-Pérot ${ }^{8,9,46}$ and photonic crystal cavities. ${ }^{4}$ Here, we achieve lasing in a dielectric BIC cavity based on inphase out-of-plane magnetic dipoles. By adjusting the diameter of the $\mathrm{TiO}_{2}$ cylinders, we tune the lasing wavelength across the gain bandwidth of the NPLs.

Figure 1a depicts a lasing device, in which a square array of $\mathrm{TiO}_{2}$ cylinders on a quartz substrate is covered with a thin film of NPLs. We fabricate a series of such $\mathrm{TiO}_{2}$ arrays, with the diameter $(D)$ of the cylinders varying from 280 to $340 \mathrm{~nm}$ across the arrays but kept constant within each array. All cylinders are $120 \mathrm{~nm}$ in height and spaced with a $40 \mathrm{~nm}$ gap in both $x$ and $y$ directions; see an SEM image in the inset of Figure 1b. We then spin-cast CdSe/CdZnS NPLs dissolved in hexane, followed by a spin-rinse with ethanol, to form a uniform film on the sample. The photoluminescence (PL) of the NPLs in hexane peaks at $\lambda$ $=642 \mathrm{~nm}$, with a full-width at half-maximum (fwhm) of $24 \mathrm{~nm}$ (Figure S1a). The PL peak red-shifts slightly in the spin-cast film to $\lambda=645 \mathrm{~nm}$ (Figure S1b). Upon spin-casting, the NPLs are deposited mainly on the top surface and side walls of the $\mathrm{TiO}_{2}$ cylinders, as suggested by the SEM image in Figure 1b. Such morphology is advantageous because the electric field is concentrated inside and on top of the Mie-resonant nanoparticles, as shown in the numerically calculated near-field intensity distribution (Figure 1c) for cylinders supporting outof-plane magnetic and electric dipole modes under BIC conditions.

We characterize the reflectance, fluorescence, and lasing properties of the sample with a customized microspectrometer with the capability of spectrally resolved back focal plane imaging. ${ }^{36}$ Measurements are performed in ambient conditions after we cover the sample with a layer of polydimethylsiloxane (PDMS), to match the refractive index of the quartz substrate. We first measure the reflectance spectra of the $\mathrm{TiO}_{2}$ arrays 

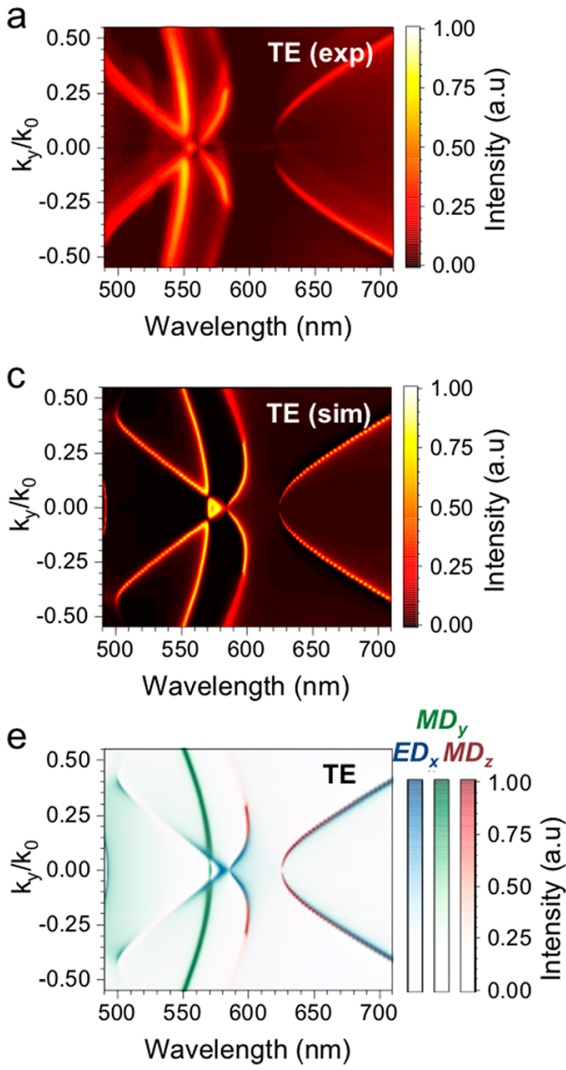
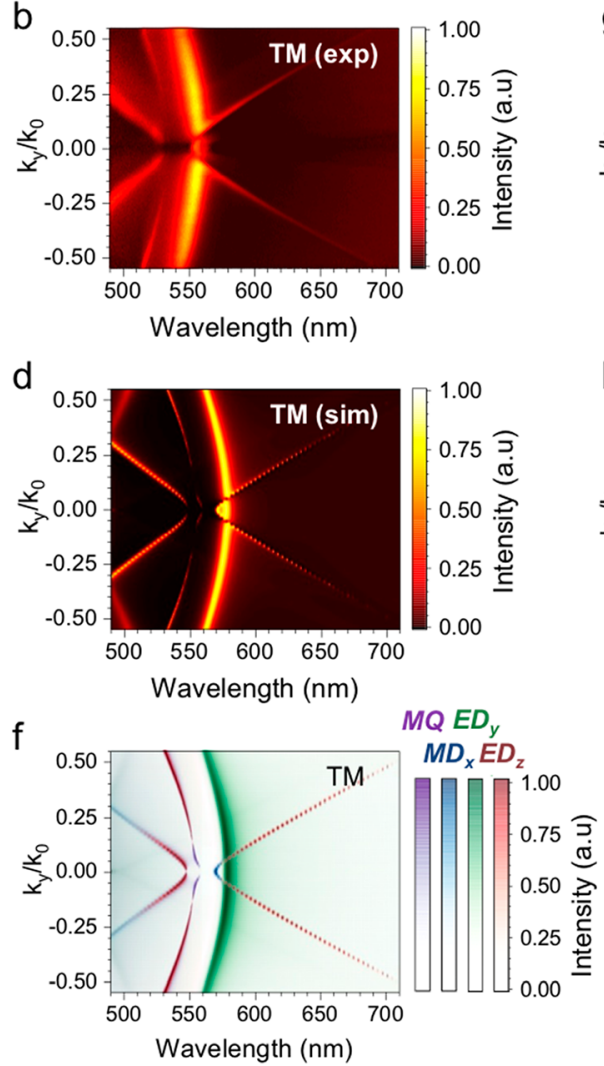
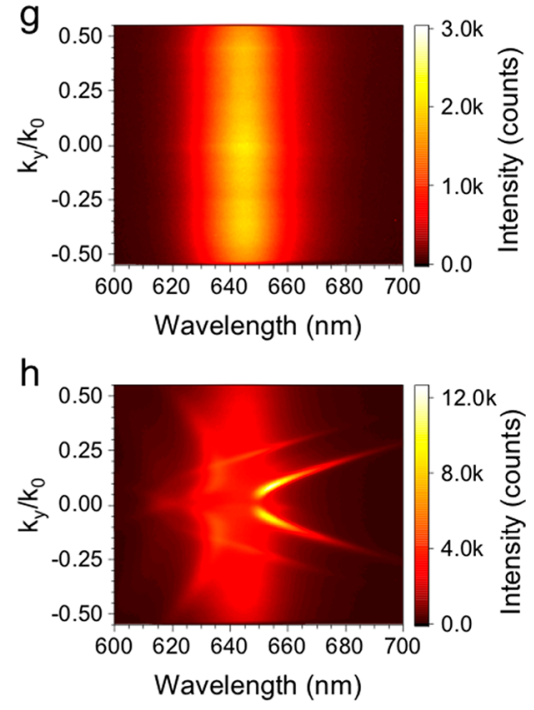

Figure 2. (a, b) Angle-resolved reflectance spectra of an array of $\mathrm{TiO}_{2}$ cylinders with diameter $\mathrm{D}=300 \mathrm{~nm}$, without nanoplatelets (NPLs), under (a) transverse electric, TE, and (b) transverse magnetic, TM, polarized light. The angle of reflection in the $y-z$ plane is denoted by the ratio between the projected in-plane momentum $k_{y}$ and free-space momentum $k_{0}$. (c, d) Numerically calculated reflectance spectra of the same array. (e, f) Corresponding multipolar decomposition revealing various Mie modes. (e) TE polarization: magnetic dipoles $\mathrm{MD}_{y}, \mathrm{MD}_{z}$ and electric dipole $\mathrm{ED}_{x}$ are excited. A BIC forms at $k_{y}=0$ from $\mathrm{MD}_{z}$ at $\lambda=625 \mathrm{~nm}$. (f) TM polarization: electric dipoles $\mathrm{ED}_{y}, \mathrm{ED}_{z}$ and magnetic dipole $\mathrm{MD}_{x}$ are excited. Two BICs form at $k_{y}=0$, from $\mathrm{ED}_{z}$ at $\lambda=548 \mathrm{~nm}$ and from a magnetic quadrupole (MQ) mode at $\lambda \sim 560 \mathrm{~nm}$, respectively. Color bars: normalized dipole moments. $(\mathrm{g}, \mathrm{h})$ Angle-resolved photoluminescence spectra of NPLs on $(\mathrm{g})$ a plain substrate and $(\mathrm{h})$ an array of TiO $\mathrm{C}_{2} \mathrm{cylinders} \mathrm{with} \mathrm{D}=300 \mathrm{~nm}$. The presence of NPLs red-shifts the modes seen in $(a-f)$.

without NPLs. Figures $2 \mathrm{a}$ and $2 \mathrm{~b}$ show the reflectance of an array with a cylinder diameter $D=300 \mathrm{~nm}$, resolved in both wavelength and angle of reflection in the $y-z$ plane, under incident light of transverse-electric (TE, Figure 2a) and transverse-magnetic (TM, Figure $2 b$ ) polarizations, with the electric field confined along the $x$ axis and in the $y-z$ plane, respectively. Numerical simulations of the reflectance (Figures $2 \mathrm{c}$ and $2 \mathrm{~d}$ ) reproduce the modes observed, and multipolar decomposition (see the Supporting Information for details) elucidates their origins (Figures $2 \mathrm{e}$ and 2f). Under TE polarization (Figures 2a, 2c, and 2e), magnetic dipoles in the $y-z$ plane $\left(\mathrm{MD}_{y}, \mathrm{MD}_{z}\right)$ and electric dipoles along $x\left(\mathrm{ED}_{x}\right)$ are excited; under TM polarization (Figures $2 \mathrm{~b}, 2 \mathrm{~d}$, and $2 \mathrm{f}$ ), electric dipoles in the $y-z$ plane $\left(\mathrm{ED}_{y}, \mathrm{ED}_{z}\right)$ and magnetic dipoles along $x\left(\mathrm{MD}_{x}\right)$ are excited. Notably, in the direction normal to the lattice plane $\left(k_{y}=0\right)$, we observe, for the TE polarization, a clear BIC based on out-of-plane magnetic dipoles $\left(\mathrm{MD}_{z}\right)$ at $\lambda \sim 625$ $\mathrm{nm}$, near the emission wavelength of the NPLs (Figures 2a, 2c, and $2 \mathrm{e}$ ). The BIC mode is characterized by spectral narrowing and vanishing reflection as the angle of incidence approaches the normal, ${ }^{36}$ as the normally incident light cannot couple to the vertically oscillating dipoles. Figure S2, derived from Figure 2a, shows a dramatic increase in the $Q$ factor of the mode as the angle of incidence (reflection) approaches zero. The maximum $Q$ factor measured, limited by the resolution of the spectrometer, is about 450 near the BIC condition (Figure S2). Similarly, for the TM polarization, two more BIC modes can be observed, based on the out-of-plane electric dipole resonance $\left(\mathrm{ED}_{z}\right)$ and a magnetic quadrupole (MQ) mode (Figures 2b, 2d, and 2f). Coating the cylinder arrays with a layer of NPLs appears to redshift the modes due to a higher refractive index of NPLs ( $n \sim$ $1.70)$ compared to PDMS $(n \sim 1.46)$ and erases some spectral features due to absorption in the NPLs (Figure S3). We note that for the array with $D=300 \mathrm{~nm}$ and with NPLs, the magnetic dipole-based BIC (MD-BIC) for the TE polarization becomes spectrally matched to the fluorescence of the NPLs.

Indeed, the PL of the NPLs shows evidence of coupling to the photonic modes of the dielectric cavity. Figures $2 \mathrm{~g}$ and $2 \mathrm{~h}$ are the angle-resolved PL spectra in the $y-z$ plane for NPLs on a plain substrate and on a $\mathrm{TiO}_{2}$ array with $D=300 \mathrm{~nm}$, respectively, when excited with a CW laser at $\lambda=488 \mathrm{~nm}$. While the $\mathrm{PL}$ of the plain area displays little angle dependence (Figure $2 \mathrm{~g}$ ), the spectrum on the array (Figure $2 \mathrm{~h}$ ) features a few bands observed in the reflectance spectra amid a background PL from uncoupled NPLs. In particular, the band associated with the $z$ oriented magnetic dipoles sees prominent enhancement, likely due to a high Purcell factor, except at the MD-BIC $\left(k_{y}=0\right)$ where radiation is inhibited as expected. Figures $S 4$ and $S 5$ show the full characterization of the PL from a series of arrays with the cylinder diameter $D$ varying from 280 to $340 \mathrm{~nm}$. Increasing $D$ red-shifts all the photonic bands (Figure S4) and selectively couples the relatively narrow PL to a subset of the modes 

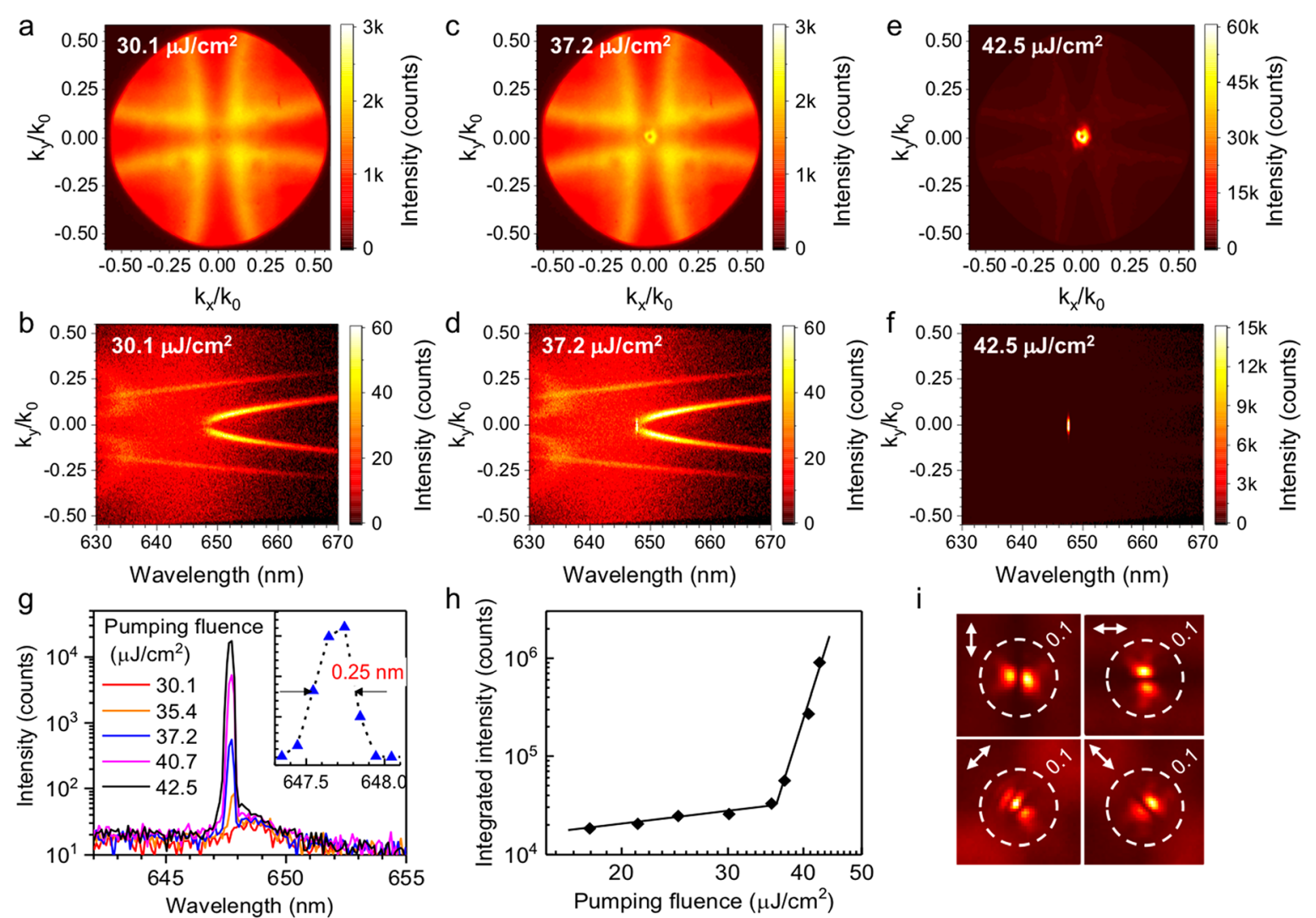

i

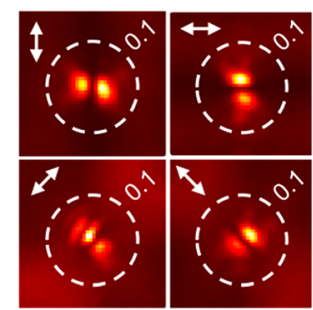

Figure 3. (a, c, e) Back focal plane (BFP) images of the nanoplatelet photoluminescence (PL) on an array of $\mathrm{TiO}_{2}$ cylinders with diameter $D=300 \mathrm{~nm}$. The array is excited with a pulsed laser at $\lambda=530 \mathrm{~nm}$ at the indicated fluences: (a) below, (c) just above, and (e) well above the lasing threshold. (b, $\mathrm{d}, \mathrm{f}$ ) Angle-resolved PL spectra obtained by spectrally resolving a thin slice of the respective BFP images (a, c, e) along $k_{x}=0$. (g) PL spectra at several pump fluences, showing the onset of lasing. Inset: spectral line width when pumped at $40.7 \mu \mathrm{J} / \mathrm{cm}^{2}$, suggesting a $Q$ factor of 2590 . (h) PL intensity as a function of the pump fluence on a log-log scale. The lasing threshold is approximately $36 \mu \mathrm{J} / \mathrm{cm}^{2}$. (i) Polarization dependence of the far-field radiation pattern for lasing. The direction of polarization of the output beam is marked by the white arrows. The dashed circle corresponds to a normalized inplane momentum $k_{||} / k_{0}$ of 0.1 .

revealed in the reflectance spectra. In addition, increasing $D$ increases the period of the lattice, thus moving the array from subdiffractive to diffractive for in-phase dipoles. This is evident from the evolution of the far-field radiation pattern on the back focal plane (Figure S5), which sees the diffraction bands of the $\mathrm{PL}$ approaching and going beyond the normal direction.

To investigate lasing, we excite the sample at $\lambda=530 \mathrm{~nm}$ with a pulsed laser (200 fs pulse width, $20 \mathrm{kHz}$ repetition rate). We study the array with $D=300 \mathrm{~nm}$ first as it shows the best spectral overlap between the MD-BIC and the PL of NPLs. As the pulse energy of excitation increases, the gain medium transitions from spontaneous emission to stimulated emission. Below the threshold, the far-field radiation pattern (Figure 3a) consists of the diffraction bands of the PL. The angle-resolved PL spectrum in the $y-z$ plane shows that the PL is primarily coupled to the out-of-plane magnetic dipoles (Figure $3 b$ ). When the pump fluence is increased just above the threshold, in addition to the diffraction bands, radiation becomes prominent in the near-normal direction (Figure 3c). A doughnut-shaped bright spot at the center of the back focal plane is observed. Spectral analysis suggests that this corresponds to the MD-BIC mode (Figure $3 \mathrm{~d}$ ). The mode exhibits a rapid gain in intensity upon further increase in the pump fluence and eventually dominates the radiation output (Figures $3 \mathrm{e}$ and $3 \mathrm{f}$ ). Spectrally, a narrow peak appears in the PL at the onset of stimulated emission (Figure $3 \mathrm{~g}$ ). The fwhm is $0.25 \mathrm{~nm}$ at a center wavelength of $647.7 \mathrm{~nm}$, subject to the resolution of the spectrometer, and thus suggests a $Q$ factor of at least 2590 for the MD-BIC cavity. The high directionality of emission, narrow spectral line width, and a clear threshold in the output-to-input power dependence (Figure $3 \mathrm{~h}$ ) are all evidence of lasing. Analysis of the doughnut-shaped radiation pattern suggests that lasing occurs at $0.8^{\circ}$ with respect to the normal into the free space (Figure S6). In addition, time-resolved PL measurement (Figure S7) proves that the rate of exciton decay is increased by an order of magnitude at lasing.

It is worth noting that the doughnut-shaped far-field radiation pattern has been observed in BIC-based lasing before. ${ }^{6,32,37,38}$ While, strictly speaking, no light should be emitted from the BIC because the mode is decoupled from the radiation field, the finite size of the sample prevents complete cancellation of radiation. In the context of phased-array antennas, a total radiation pattern can be derived from the pattern of each individual element modulated by an array factor. ${ }^{47,48}$ For in-phase emitters in an infinite subdiffractive array, the array factor is nonzero only in the normal direction. When the array is finite in size, however, the factor acquires a certain angular width around the normal direction, allowing some radiation to escape, which ultimately limits the $Q$ factor of the quasi-BIC mode. The leaked radiation should bear the polarization characteristics of the radiation of each constituent element in the array. Indeed, the measured polarization dependence of the lasing output (Figure 3i) reflects the TE-polarized nature of the radiation from $z$-oriented 

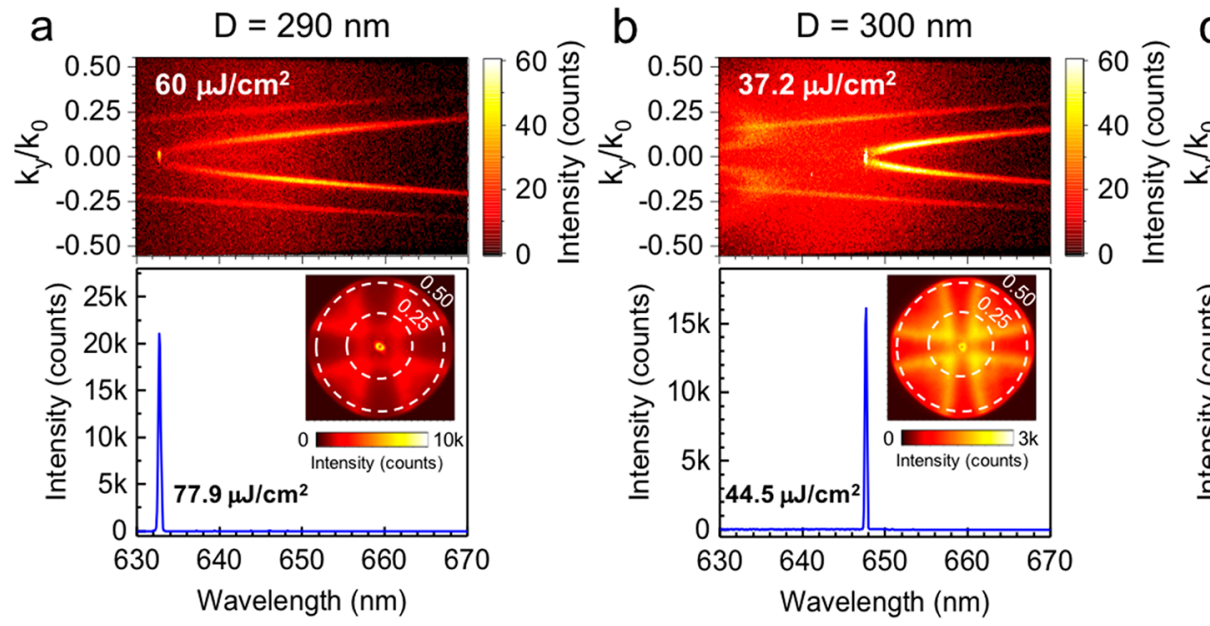

C
0.50
0.25
$\times-0.00$
-0.25
-0.50

$\mathrm{D}=310 \mathrm{~nm}$

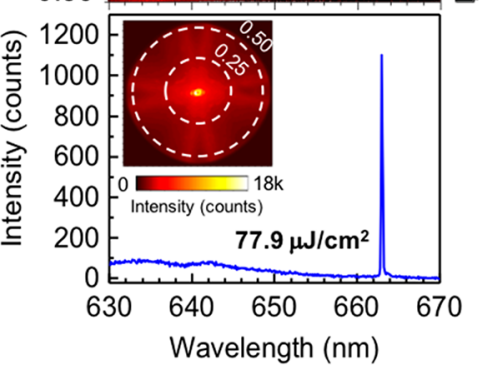

Figure 4. $(\mathrm{a}-\mathrm{c})$ Upper panel: angle-resolved photoluminescence (PL) spectra of nanoplatelets on three arrays of $\mathrm{TiO}_{2}$ cylinders with diameters (a) $D$ $=290 \mathrm{~nm},(\mathrm{~b}) D=300 \mathrm{~nm}$, and (c) $D=310 \mathrm{~nm}$. The arrays are excited at the indicated fluences, just above their respective lasing thresholds. Increasing the diameter of $\mathrm{TiO}_{2}$ cylinders red-shifts the MD-BIC mode responsible for lasing. Inset of lower panel: back focal plane images of the PL at the onset of lasing. All show a doughnut-shaped radiation pattern in the normal direction attributed to the MD-BIC. Dashed circles are labeled with normalized in-plane momentum $k_{\|} / k_{0}$. Lower panel: lasing spectra measured at the indicated pump fluences, which are comfortably above the respective lasing thresholds.

magnetic dipoles and matches the simulation results (Figure S8), confirming that lasing is realized via the MD-BIC.

Besides the array with cylinder diameter $D=300 \mathrm{~nm}$, we also observe lasing from arrays with $D=290 \mathrm{~nm}$ and $D=310 \mathrm{~nm}$, albeit at higher thresholds. Figures $4 a-c$ compare the angleresolved PL spectrum, far-field radiation pattern, and lasing wavelength for the three arrays. The PL spectra (upper panels of Figures $4 a-c$ ) and the radiation patterns (insets in the lower panels of Figures $4 a-c$ ), both measured at the onset of lasing, show that lasing originates from the same MD-BIC mode, which red-shifts in wavelength as $D$ increases. The mode is situated optimally in the middle of the NPL gain bandwidth for $D=300$ $\mathrm{nm}$, while it is located at the blue and red edges of the gain bandwidth for $D=290 \mathrm{~nm}$ and $D=310 \mathrm{~nm}$, respectively, which explains why the lasing threshold is the lowest for $D=300 \mathrm{~nm}$. The lasing spectra measured at pump fluences well above the threshold (lower panels of Figures $4 \mathrm{a}-\mathrm{c}$ ) show an approximately $30 \mathrm{~nm}$ red-shift in the lasing wavelength from $D=290 \mathrm{~nm}$ to $D=$ $310 \mathrm{~nm}$. The ability to tune the lasing wavelength by changing the physical dimensions of the dielectric nanoresonators, together with the spectral tunability of the gain of NPLs, makes a wide range of lasing wavelengths easily accessible.

Finally, at an even higher threshold, we observe lasing from the array with cylinder diameter $D=340 \mathrm{~nm}$. Full characterization (Figure S9) reveals that lasing occurs from a BIC comprising resonant magnetic quadrupoles (MQ-BIC). The farfield radiation pattern sees the emergence of intense emission near the normal direction, and when zoomed in, it consists of four bright lobes with nodes along $x, y$, and $z$ directions (Figure S9c), instead of being doughnut-shaped as in MD-BIC. This suggests a quadrupole-based BIC, which is found to be a magnetic quadrupole mode in the multipolar decomposition (Figure S9j). We attribute the higher lasing threshold from the MQ-BIC, compared to MD-BIC, to poorer spectral overlap with the NPL gain and a lower $Q$ factor. The latter is caused by diffractive losses in the array which has a period large enough for radiation outcoupling at oblique angles of in-phase multipoles.

In summary, we demonstrate optically pumped roomtemperature lasing in a solution-processed $\mathrm{CdSe} / \mathrm{CdZnS} \mathrm{NPL}$ film with an all-dielectric cavity supporting BICs. The BICs arise from lattice-mediated Mie resonances in $\mathrm{TiO}_{2}$ nanocylinders and possess high $Q$ factors. We achieve lasing from a BIC that originates from a subdiffractive array of out-of-plane magnetic dipoles oscillating in phase, as well as a BIC based on a diffractive array of in-phase magnetic quadrupoles. Within the gain bandwidth of the NPLs, we show tunability in the lasing wavelength by changing the size of the $\mathrm{TiO}_{2}$ cylinders. Given that the gain of NPLs can be tuned spectrally by their thickness and chemical composition, a wide selectivity in the lasing wavelength is expected in such a hybrid device structure. This offers unprecedented freedom and convenience on the design and implementation of lasing devices based on dielectric Mie resonances, particularly in cavities that support high-Q BICs, and should lead to more efficient, lower threshold, longer pulsed, or CW lasers in a wide range of lasing wavelengths in the future.

\section{ASSOCIATED CONTENT}

St Supporting Information

The Supporting Information is available free of charge at https://pubs.acs.org/doi/10.1021/acs.nanolett.0c01975.

Methods; absorption and photoluminescence (PL) spectra of colloidal nanoplatelets (NPLs); Q factor of MD-BIC in an array of $\mathrm{TiO}_{2}$ cylinders without NPLs; angle-resolved reflectance spectra, PL spectra, and back focal plane PL images of NPL-covered arrays with various cylinder diameters; angle distribution of the lasing radiation; comparison of PL lifetimes below and above the lasing threshold; simulated polarization dependence of the far-field radiation pattern for lasing; and back focal plane images and PL spectra for lasing from MQ-BIC in an array with cylinder diameter $D=340 \mathrm{~nm}$ (PDF)

\section{AUTHOR INFORMATION}

\section{Corresponding Authors}

Arseniy I. Kuznetsov - Institute of Materials Research and Engineering, A*STAR (Agency for Science, Technology and Research), Singapore 138634, Singapore; 이이이.org/0000- 
0002-7622-8939; Email: arseniy_kuznetsov@imre.astar.edu.sg

Hilmi Volkan Demir - LUMINOUS! Centre of Excellence for Semiconductor Lighting and Displays, Nanyang Technological University, Singapore 639798, Singapore; UNAM-Institute of Materials Science and Nanotechnology, Bilkent University, 06800 Ankara, Turkey; (이이.org/0000-0003-1793-112X; Email: hvdemir@ntu.edu.sg

\section{Authors}

Mengfei Wu - Institute of Materials Research and Engineering, A*STAR (Agency for Science, Technology and Research), Singapore 138634, Singapore; 이이.org/0000-0002-47283234

Son Tung Ha - Institute of Materials Research and Engineering, A*STAR (Agency for Science, Technology and Research), Singapore 138634, Singapore; 이이.org/0000-0002-54758365

Sushant Shendre - LUMINOUS! Centre of Excellence for Semiconductor Lighting and Displays, Nanyang Technological University, Singapore 639798, Singapore

Emek G. Durmusoglu - LUMINOUS! Centre of Excellence for Semiconductor Lighting and Displays, Nanyang Technological University, Singapore 639798, Singapore; 이이이.org/00000001-6840-8342

Weon-Kyu Koh - LUMINOUS! Centre of Excellence for Semiconductor Lighting and Displays, Nanyang Technological University, Singapore 639798, Singapore

Diego R. Abujetas - Instituto de Estructura de la Materia (IEMCSIC), Consejo Superior de Investigaciones Cientificas, 28006 Madrid, Spain; 이이이.org/0000-0002-6544-5305

José A. Sánchez-Gil - Instituto de Estructura de la Materia (IEM-CSIC), Consejo Superior de Investigaciones Cientificas, 28006 Madrid, Spain; 이이.org/0000-0002-5370-3717

Ramón Paniagua-Domínguez - Institute of Materials Research and Engineering, A*STAR (Agency for Science, Technology and Research), Singapore 138634, Singapore; 이잉.org/00000001-7836-681X

Complete contact information is available at:

https://pubs.acs.org/10.1021/acs.nanolett.0c01975

\section{Author Contributions}

${ }^{\perp}$ M.W. and S.T.H. contributed equally to this work. A.I.K., S.T.H., and H.V.D. conceived the idea. M.W. fabricated the samples, measured reflectance, and wrote the first draft of the manuscript. S.T.H. performed optical characterization on the lasing sample and prepared the figures. S.S., E.G.D., and W.K.K. synthesized and characterized the nanoplatelets with inputs from H.V.D. S.S. prepared the nanoplatelet solutions and performed spin-cast deposition. R.P.-D., D.R.A., and J.A.S.-G. performed the numerical simulations and theoretical analysis. H.V.D. and A.I.K. supervised the project. All authors contributed to writing the manuscript.

\section{Notes}

The authors declare no competing financial interest.

\section{ACKNOWLEDGMENTS}

This work was supported by the A*STAR SERC Pharos programme (grant number 15273 00025; Singapore). D.R.A. and J.A.S.-G. acknowledge support from the Spanish Ministerio de Ciencia e Innovación (NANOTOPO FIS2017-91413-EXP, MELODIA PGC2018-095777-B-C21, and a FPU Ph.D. Fellow- ship FPU15/03566, MCIU/AEI/FEDER, UE). H.V.D. gratefully acknowledges support from TUBA. The authors acknowledge Vytautas Valuckas (IMRE, A*STAR) for SEM characterization.

\section{REFERENCES}

(1) Hill, M. T.; Gather, M. C. Advances in Small Lasers. Nat. Photonics 2014, 8, 908-918.

(2) Ma, R.-M.; Oulton, R. F. Applications of Nanolasers. Nat. Nanotechnol. 2019, 14, 12-22.

(3) Fan, F.; Voznyy, O.; Sabatini, R. P.; Bicanic, K. T.; Adachi, M. M.; McBride, J. R.; Reid, K. R.; Park, Y. S.; Li, X.; Jain, A.; et al. ContinuousWave Lasing in Colloidal Quantum Dot Solids Enabled by FacetSelective Epitaxy. Nature 2017, 544, 75-79.

(4) Yang, Z.; Pelton, M.; Fedin, I.; Talapin, D. V.; Waks, E. A Room Temperature Continuous-Wave Nanolaser Using Colloidal Quantum Wells. Nat. Commun. 2017, 8, 143.

(5) Zhou, W.; Dridi, M.; Suh, J.Y.; Kim, C. H.; Co, D. T.; Wasielewski, M. R.; Schatz, G. C.; Odom, T. W. Lasing Action in Strongly Coupled Plasmonic Nanocavity Arrays. Nat. Nanotechnol. 2013, 8, 506-511.

(6) Hakala, T. K.; Rekola, H. T.; Väkeväinen, A. I.; Martikainen, J. P.; Nečada, M.; Moilanen, A. J.; Törmä, P. Lasing in Dark and Bright Modes of a Finite-Sized Plasmonic Lattice. Nat. Commun. 2017, 8, 13687.

(7) Dang, C.; Lee, J.; Breen, C.; Steckel, J. S.; Coe-Sullivan, S.; Nurmikko, A. Red, Green and Blue Lasing Enabled by Single-Exciton Gain in Colloidal Quantum Dot Films. Nat. Nanotechnol. 2012, 7, 335339.

(8) Grim, J. Q.; Christodoulou, S.; Di Stasio, F.; Krahne, R.; Cingolani, R.; Manna, L.; Moreels, I. Continuous-Wave Biexciton Lasing at Room Temperature Using Solution-Processed Quantum Wells. Nat. Nanotechnol. 2014, 9, 891-895.

(9) Guzelturk, B.; Kelestemur, Y.; Olutas, M.; Delikanli, S.; Demir, H. V. Amplified Spontaneous Emission and Lasing in Colloidal Nanoplatelets. ACS Nano 2014, 8, 6599-6605.

(10) Bulović, V.; Kozlov, V. G.; Khalfin, V. B.; Forrest, S. R. Transform-Limited, Narrow-Linewidth Lasing Action in Organic Semiconductor Microcavities. Science 1998, 279, 553-555.

(11) Kravets, V. G.; Schedin, F.; Grigorenko, A. N. Extremely Narrow Plasmon Resonances Based on Diffraction Coupling of Localized Plasmons in Arrays of Metallic Nanoparticles. Phys. Rev. Lett. 2008, 101, 087403.

(12) Auguié, B.; Barnes, W. L. Collective Resonances in Gold Nanoparticle Arrays. Phys. Rev. Lett. 2008, 101, 143902.

(13) Vecchi, G.; Giannini, V.; Gómez Rivas, J. Shaping the Fluorescent Emission by Lattice Resonances in Plasmonic Crystals of Nanoantennas. Phys. Rev. Lett. 2009, 102, 146807.

(14) Lozano, G.; Grzela, G.; Verschuuren, M. A.; Ramezani, M.; Rivas, J. G. Tailor-Made Directional Emission in Nanoimprinted PlasmonicBased Light-Emitting Devices. Nanoscale 2014, 6, 9223-9229.

(15) Kuznetsov, A. I.; Miroshnichenko, A. E.; Brongersma, M. L.; Kivshar, Y. S.; Luk'yanchuk, B. Optically Resonant Dielectric Nanostructures. Science 2016, 354, aag2472.

(16) Staude, I.; Schilling, J. Metamaterial-Inspired Silicon Nanophotonics. Nat. Photonics 2017, 11, 274-284.

(17) Paniagua-Dominguez, R.; Ha, S. T.; Kuznetsov, A. I. Active and Tunable Nanophotonics With Dielectric Nanoantennas. Proc. IEEE 2020, 108, 749-771.

(18) Kuznetsov, A. I.; Miroshnichenko, A. E.; Fu, Y. H.; Zhang, J.; Luk'yanchuk, B. Magnetic Light. Sci. Rep. 2012, 2, 492.

(19) Evlyukhin, A. B.; Novikov, S. M.; Zywietz, U.; Eriksen, R. L.; Reinhardt, C.; Bozhevolnyi, S. I.; Chichkov, B. N. Demonstration of Magnetic Dipole Resonances of Dielectric Nanospheres in the Visible Region. Nano Lett. 2012, 12, 3749-3755.

(20) Evlyukhin, A. B.; Reinhardt, C.; Seidel, A.; Luk'Yanchuk, B. S.; Chichkov, B. N. Optical Response Features of Si-Nanoparticle Arrays. Phys. Rev. B: Condens. Matter Mater. Phys. 2010, 82, 045404. 
(21) Babicheva, V. E.; Evlyukhin, A. B. Resonant Lattice Kerker Effect in Metasurfaces With Electric and Magnetic Optical Responses. Laser Photonics Rev. 2017, 11, 1700132.

(22) Hsu, C. W.; Zhen, B.; Stone, A. D.; Joannopoulos, J. D.; Soljačić, M. Bound States in the Continuum. Nat. Rev. Mater. 2016, 1, 16048.

(23) Koshelev, K.; Favraud, G.; Bogdanov, A.; Kivshar, Y.; Fratalocchi, A. Nonradiating Photonics with Resonant Dielectric Nanostructures. Nanophotonics 2019, 8, 725-745.

(24) Sadrieva, Z.; Frizyuk, K.; Petrov, M.; Kivshar, Y.; Bogdanov, A. Multipolar Origin of Bound States in the Continuum. Phys. Rev. B: Condens. Matter Mater. Phys. 2019, 100, 115303.

(25) Abujetas, D. R.; Barreda, Á.; Moreno, F.; Sáenz, J. J.; Litman, A.; Geffrin, J.-M.; Sánchez-Gil, J. A. Brewster Quasi Bound States in the Continuum in All-Dielectric Metasurfaces from Single MagneticDipole Resonance Meta-Atoms. Sci. Rep. 2019, 9, 16048.

(26) Sadrieva, Z. F.; Sinev, I. S.; Koshelev, K. L.; Samusev, A.; Iorsh, I. V.; Takayama, O.; Malureanu, R.; Bogdanov, A. A.; Lavrinenko, A. V. Transition from Optical Bound States in the Continuum to Leaky Resonances: Role of Substrate and Roughness. ACS Photonics 2017, 4, $723-727$.

(27) Koshelev, K.; Lepeshov, S.; Liu, M.; Bogdanov, A.; Kivshar, Y. Asymmetric Metasurfaces with High- Q Resonances Governed by Bound States in the Continuum. Phys. Rev. Lett. 2018, 121, 193903.

(28) Campione, S.; Liu, S.; Basilio, L. I.; Warne, L. K.; Langston, W. L.; Luk, T. S.; Wendt, J. R.; Reno, J. L.; Keeler, G. A.; Brener, I.; et al. Broken Symmetry Dielectric Resonators for High Quality Factor Fano Metasurfaces. ACS Photonics 2016, 3, 2362-2367.

(29) Abujetas, D. R.; van Hoof, N.; ter Huurne, S.; Gómez Rivas, J.; Sánchez-Gil, J. A. Spectral and Temporal Evidence of Robust Photonic Bound States in the Continuum on Terahertz Metasurfaces. Optica 2019, 6, 996-1001.

(30) Yuan, S.; Qiu, X.; Cui, C.; Zhu, L.; Wang, Y.; Li, Y.; Song, J.; Huang, Q.; Xia, J. Strong Photoluminescence Enhancement in AllDielectric Fano Metasurface with High Quality Factor. ACS Nano 2017, 11, 10704-10711.

(31) Liu, S.; Vaskin, A.; Addamane, S.; Leung, B.; Tsai, M. C.; Yang, Y.; Vabishchevich, P. P.; Keeler, G. A.; Wang, G.; He, X.; et al. LightEmitting Metasurfaces: Simultaneous Control of Spontaneous Emission and Far-Field Radiation. Nano Lett. 2018, 18, 6906-6914.

(32) Miyai, E.; Sakai, K.; Okano, T.; Kunishi, W.; Ohnishi, D.; Noda, S. Lasers Producing Tailored Beams. Nature 2006, 441, 946.

(33) Zhen, B.; Chua, S.-L.; Lee, J.; Rodriguez, A. W.; Liang, X.; Johnson, S. G.; Joannopoulos, J. D.; Soljačić, M.; Shapira, O. Enabling Enhanced Emission and Low-Threshold Lasing of Organic Molecules Using Special Fano Resonances of Macroscopic Photonic Crystals. Proc. Natl. Acad. Sci. U. S. A. 2013, 110, 13711-13716.

(34) Hirose, K.; Liang, Y.; Kurosaka, Y.; Watanabe, A.; Sugiyama, T.; Noda, S. Watt-Class High-Power, High-Beam-Quality PhotonicCrystal Lasers. Nat. Photonics 2014, 8, 406-411.

(35) Kodigala, A.; Lepetit, T.; Gu, Q.; Bahari, B.; Fainman, Y.; Kante, B. Lasing Action from Photonic Bound States in Continuum. Nature 2017, 541, 196-199.

(36) Ha, S. T.; Fu, Y. H.; Emani, N. K.; Pan, Z.; Bakker, R. M.; Paniagua-Domínguez, R; Kuznetsov, A. I. Directional Lasing in Resonant Semiconductor Nanoantenna Arrays. Nat. Nanotechnol. 2018, 13, 1042-1047.

(37) Huang, C.; Zhang, C.; Xiao, S.; Wang, Y.; Fan, Y.; Liu, Y.; Zhang, N.; Qu, G.; Ji, H.; Han, J.; et al. Ultrafast Control of Vortex Microlasers. Science 2020, 367, 1018-1021.

(38) Van Beijnum, F.; Van Veldhoven, P. J.; Geluk, E. J.; De Dood, M. J. A.; 't Hooft, G. W.; Van Exter, M. P. Surface Plasmon Lasing Observed in Metal Hole Arrays. Phys. Rev. Lett. 2013, 110, 206802.

(39) Ramezani, M.; Halpin, A.; Fernández-Domínguez, A. I.; Feist, J.; Rodriguez, S. R.-K.; Garcia-Vidal, F. J.; Gómez Rivas, J. PlasmonExciton-Polariton Lasing. Optica 2017, 4, 31-37.

(40) Guan, J.; Sagar, L. K.; Li, R.; Wang, D.; Bappi, G.; Wang, W.; Watkins, N.; Bourgeois, M. R.; Levina, L.; Fan, F.; et al. Quantum DotPlasmon Lasing with Controlled Polarization Patterns. ACS Nano 2020, 14, 3426-3433.
(41) Guan, J.; Sagar, L. K.; Li, R.; Wang, D.; Bappi, G.; Watkins, N. E.; Bourgeois, M. R.; Levina, L.; Fan, F.; Hoogland, S.; et al. Engineering Directionality in Quantum Dot Shell Lasing Using Plasmonic Lattices. Nano Lett. 2020, 20, 1468-1474.

(42) Ithurria, S.; Tessier, M. D.; Mahler, B.; Lobo, R. P. S. M.; Dubertret, B.; Efros, A. L. Colloidal Nanoplatelets with TwoDimensional Electronic Structure. Nat. Mater. 2011, 10, 936-941.

(43) Antanovich, A.; Prudnikau, A.; Matsukovich, A.; Achtstein, A.; Artemyev, M. Self-Assembly of CdSe Nanoplatelets into Stacks of Controlled Size Induced by Ligand Exchange. J. Phys. Chem. C 2016, 120, 5764-5775.

(44) Altintas, Y.; Gungor, K.; Gao, Y.; Sak, M.; Quliyeva, U.; Bappi, G.; Mutlugun, E.; Sargent, E. H.; Demir, H. V. Giant Alloyed Hot Injection Shells Enable Ultralow Optical Gain Threshold in Colloidal Quantum Wells. ACS Nano 2019, 13, 10662-10670.

(45) Pelton, M. Carrier Dynamics, Optical Gain, and Lasing with Colloidal Quantum Wells. J. Phys. Chem. C 2018, 122, 10659-10674.

(46) She, C.; Fedin, I.; Dolzhnikov, D. S.; Dahlberg, P. D.; Engel, G. S.; Schaller, R. D.; Talapin, D. V. Red, Yellow, Green, and Blue Amplified Spontaneous Emission and Lasing Using Colloidal CdSe Nanoplatelets. ACS Nano 2015, 9, 9475-9485.

(47) Orphanidis, S. J. Electromagnetic Waves and Antennas; Rutgers University: 2016; http://www.ece.rutgers.edu/ orfanidi/ewa.

(48) Paniagua-Domínguez, R.; Yu, Y. F.; Miroshnichenko, A. E.; Krivitsky, L. A.; Fu, Y. H.; Valuckas, V.; Gonzaga, L.; Toh, Y. T.; Kay, A. Y. S.; Lukyanchuk, B.; et al. Generalized Brewster Effect in Dielectric Metasurfaces. Nat. Commun. 2016, 7, 10362. 